Rev Inv Vet Perú 2015; 26(3): 537-542

http://dx.doi.org/10.15381/rivep.v26i3.11180

\title{
Comunicación
}

\section{Insuficiencia Luteal en una Perra: Descripción de un Caso}

\author{
Luteal Insufficiency in a Bitch: A CaSe Description
}

Alfonso Sánchez R. ${ }^{1,2}$

\section{Resumen}

Se describe y discute el caso de una perra Bulldog Francés de 2 años y 3 meses de edad, con una gestación de aproximadamente 37 días, con sangrado vaginal por 12 días. Mediante examen serológico se descartó la posibilidad de aborto infeccioso (B. canis) y al evaluar progesterona sérica, mediante kit comercial, se observó un valor disminuido (2.5-5 ng/ml), por lo cual se instauró una terapia de soporte mediante progesterona micronizada $(100 \mathrm{mg} /$ día $)$ hasta observar el cese del sangrado y mantención normal de la preñez. La evaluación de progesterona a partir de ese momento presentó valores mayores de $5 \mathrm{ng} / \mathrm{ml}$. Luego del día 42 de gestación, la suplementación hormonal se modificó a 100 mg cada 48 horas y se suspendió el día 58 de preñez. La hembra parió un cachorro vivo y otros tres fueron extraídos mediante operación cesárea (2 vivos y 1 muerto).

Palabras clave: insuficiencia luteal, progesterona, perra

\section{Abstract}

The present report describes and discuss the case of a French bulldog bitch, 2 years and 3 months old with a gestation of about 37 days and vaginal bleeding for nearly 12 days. Infection by $B$. canis was discarded by serological examination. Determination of serum progesterone through commercial kit showed a low value $(2.5-5 \mathrm{ng} / \mathrm{ml})$ so supportive therapy was instituted by micronized progesterone (100 $\mathrm{mg} /$ day) until the cessation of vaginal bleeding and maintenance of pregnancy. Progesterone value at this point showed higher values ( $>5 \mathrm{ng} / \mathrm{ml})$. After day 42 of gestation, hormone supplementation was modified to $100 \mathrm{mg}$ every 48 hours and stopped on day 58 of pregnancy. The female gave birth to a live puppy and other three were removed by cesarean section (two alive and one dead).

Key words: luteal insufficiency, progesterone, bitch

\footnotetext{
${ }^{1}$ Escuela de Medicina Veterinaria, Universidad Santo Tomás, Viña del Mar, Chile

${ }^{2}$ E-mail: teriogenologiachile@vtr.net
}

Recibido: 24 de diciembre de 2014

Aceptado para publicación: 1 de abril de 2015 


\section{INTRODUCCIÓN}

La perra doméstica (Canis lupus familiaris) es una hembra monoéstrica, no estacional, politoca, ovuladora espontánea, con una fase luteal de aproximadamente dos meses de duración, seguida de un anestro obligatorio hasta el siguiente periodo de calor. El intervalo interestral (IIE) fluctúa entre los cinco y doce meses no presentando diferencias significativas entre hembras gestantes y no gestantes (Concannon, 2011).

A diferencia de las especies de mamíferos de granja, en las cuales la ciclicidad depende de la producción y secreción periódica de prostaglandina $\mathrm{F}_{2 \alpha}\left(\mathrm{PGF}_{2 \alpha}\right)$ por el endometrio, la función luteal en perras no gestantes es independiente de luteolisinas uterinas. Esto ha sido demostrado en hembras histerectomizadas donde la regresión luteal ocurrió sin necesidad de la secreción de PGF $_{2 \alpha}$ uterina (Hoffmann et al., 1992). Dada la similitud en la duración y comportamiento de la producción de progesterona $\left(\mathrm{P}_{4}\right)$ entre perras en diestro y perras preñadas, se puede señalar que la vida útil fisiológica y los mecanismos de control endocrino de los cuerpos lúteos caninos son similares (Kowalewski, 2014). Asimismo, cabe destacar que la mantención de la gestación canina es dependiente de $\mathrm{P}_{4}$ y que la hormona circulante durante toda la gestación es de origen luteal. Los niveles séricos de $\mathrm{P}_{4}$ alcanzan un pico de $15-80 \mathrm{ng} / \mathrm{ml}$ entre los días 20 y 35 después del pico de hormona luteinizante (LH) para luego declinar lentamente y alcanzar un valor inferior a $2 \mathrm{ng} / \mathrm{ml}$ al momento del parto (Concannon, 2011).

La insuficiencia luteal o hipoluteinismo se caracteriza por una disminución prematura en las concentraciones séricas de $\mathrm{P}_{4}$, generando la pérdida de la gestación. Esta condición es un posible diagnóstico para pérdidas gestacionales (reabsorción, aborto, parto prematuro) en ausencia de causas infecciosas o anormalidades no infecciosas visibles (Johnston et al., 2001). Concentraciones séricas de $\mathrm{P}_{4}$ menores de $5 \mathrm{ng} / \mathrm{ml}$ en la cuarta a quinta semana de gestación sugieren insuficiencia luteal o hipoluteinismo. La insuficiencia luteal en la perra se describe comúnmente durante la segunda mitad de la gestación y es acompañada de un acortamiento del ciclo estral (Root Kustritz, 2001). Obtener un diagnóstico claro de la insuficiencia luteal a menudo es complicado, ya que la declinación de los niveles séricos de $\mathrm{P}_{4}$ podría estar asociado de forma secundaria a muerte fetal o a influencia patológica de agentes infecciosos como B. canis, E. coli o Streptococcus sp (Verstegen et al., 2008).

\section{Caso Clínico}

Una hembra Bulldog Francés, nulípara, de dos años y tres meses, peso corporal de $9.6 \mathrm{~kg}$, fue llevada el 12 de noviembre de 2014 a consulta de especialidad al Hospital Veterinario de la Universidad Santo Tomás en Viña del Mar, Chile, por presentar secreción vaginal sanguinolenta abundante por 12 días. La hembra había sido inseminada el 4 y 6 de octubre, y tenía un diagnóstico ecográfico positivo de gestación realizado el 30 de octubre.

En el examen clínico general, las constantes fisiológicas se encontraron dentro de los rangos normales para la especie. Dadas las características del cuadro y la sospecha de posible aborto, se realizó ecografía, citología vaginal y se tomaron muestras sanguíneas de la vena cefálica para evaluación de anticuerpos contra Brucella canis y determinación de $\mathrm{P}_{4}$.

En la evaluación ecográfica del latido cardiaco fetal y del diámetro biparietal, se constató una gestación de cuatro fetos vivos con una edad de $37 \pm 3$ días. En la citología vaginal se observó abundante cantidad de eritrocitos, escasos neutrófilos y predominio de células vaginales intermedias. La evaluación de anticuerpos para $B$. canis se practicó con un kit comercial (Anigen Rapid C. Brucella Ab Test Kit, BioNote, Rep. de Corea) dando un resultado negativo. La determinación de $\mathrm{P}_{4}$ sérica, se realizó utilizando un kit 


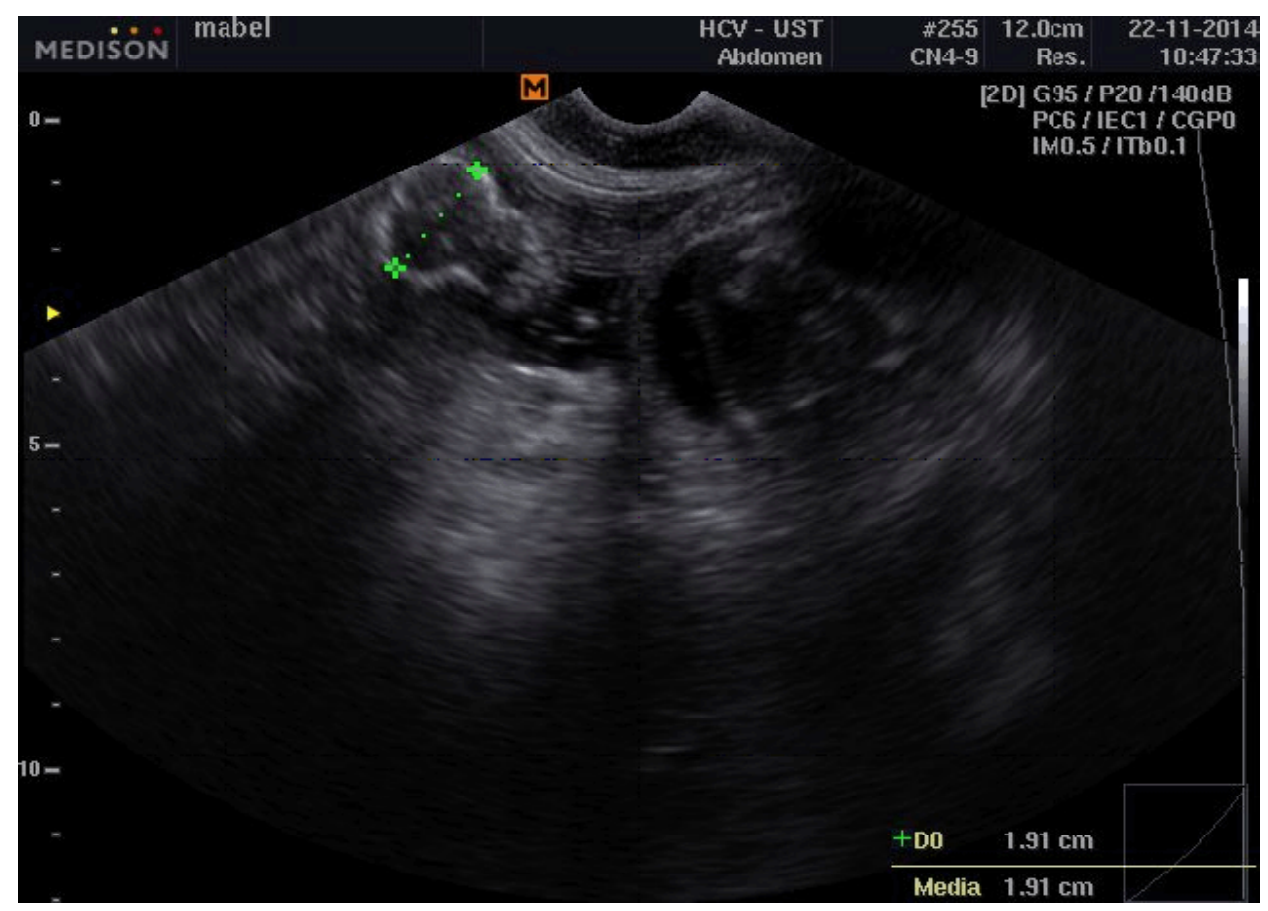

Figura 1. Ecografía gestacional de una perra Bulldog Francés con aproximadamente 48 días de gestación y luego de 11 días de suplementación con progesterona oral

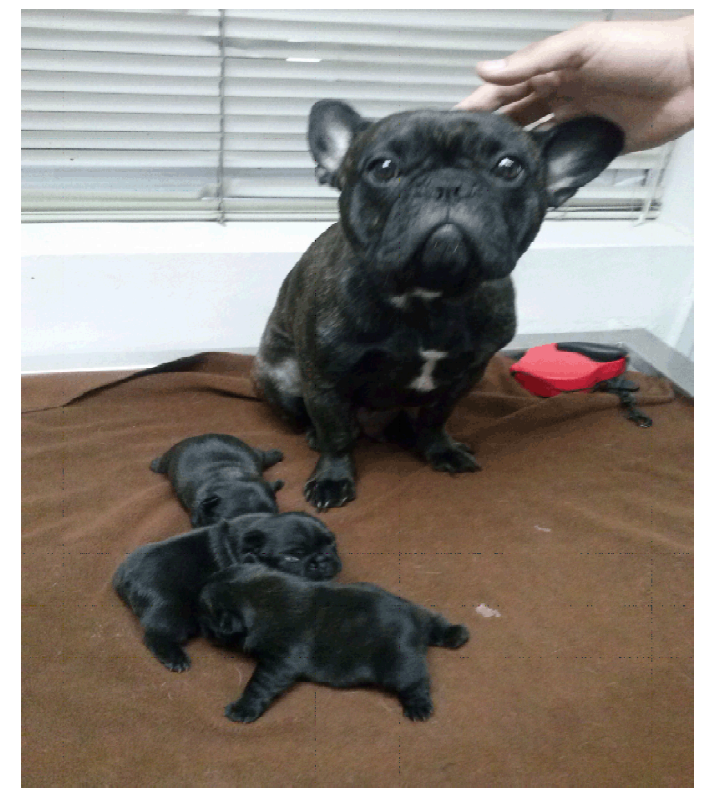

Figura 2. Hembra con sus tres cachorros de 12 días de edad comercial semicuantitativo (Target Canine Ovulation Timing kit, BioMetallics, EEUU) resultando un valor entre 2.5 y $5 \mathrm{ng} / \mathrm{ml}$. En consideración al bajo nivel de $\mathrm{P}_{4}$ se decide instaurar una terapia de soporte mediante la administración de progesterona micronizada oral (Progendo®, Recalcine, Chile), en dosis de $100 \mathrm{mg} /$ día, y proceder a evaluaciones regulares para observar el progreso de la gestación.

El propietario indicó que el sangrado vaginal dejó de observarse al cuarto día de tratamiento. $\mathrm{Al}$ día siguiente (17 de noviembre) se realiza un control ecográfico, citológico y evaluación de $\mathrm{P}_{4}$ con la metodología antes descrita. Ecográficamente se apreció una gestación normal de cuatro fetos vivos con una edad de $40 \pm 3$ días; la citología vaginal destaca la ausencia de eritrocitos y otras cé- 
lulas sanguíneas y la $\mathrm{P}_{4}$ sérica arroja un valor $>5 \mathrm{ng} / \mathrm{ml}$. En esta oportunidad se indica modificar el ritmo de administración de la progesterona micronizada a $100 \mathrm{mg}$ cada 48 horas. Se realiza otro control el 22 de noviembre confirmándose que la gestación proseguía su cuso normal (Fig. 1) y la $\mathrm{P}_{4}$ sérica se mantenía en un valor $>5 \mathrm{ng} / \mathrm{ml}$.

El 2 de diciembre se realiza un último control ecográfico para estimar la madurez fetal y la posibilidad de realizar una cesárea. Se observan fetos con frecuencia cardíaca promedio de 236 latidos por minuto y aun sin signos orgánicos de madurez como la visualización de intestinos (Linde-Forsberg, 2010). Se indica suspender la administración de progesterona micronizada y comenzar a controlar temperatura rectal en la hembra. El 4 de diciembre, el propietario informa un registro de temperatura rectal de $36.9^{\circ} \mathrm{C}$ y se le se indica llevar a la paciente para la realización de la cesárea. Un cachorro nació mientras se preparaba a la hembra para el acto quirúrgico. De los tres restantes, uno falleció en el canal de parto a causa de una alteración en la estática fetal. Tres cachorros se encuentran vivos (Fig. 2).

\section{Discusión}

El presente reporte presenta el caso de una hembra Bulldog Francés, nulípara, con cinco semanas de gestación y con un profuso sangrado vaginal por casi dos semanas. En primera instancia, cabe considerar que existen múltiples factores que pueden ocasionar pérdidas gestacionales en la perra; dentro de estos, los más destacados son los de tipo infeccioso como B. canis o factores maternos como enfermedad sistémica (Lamm y Makloski, 2012). Al resultar negativo a anticuerpos contra $B$. canis, el siguiente punto a analizar resultó ser el bajo nivel de $\mathrm{P}_{4}$ sérica, el cual se encontraba en el límite de lo aceptado como adecuado para la mantención de la gestación en la perra $(2.5-5 \mathrm{ng} / \mathrm{ml})$ (Root
Kustritz, 2001; Concannon, 2011). Este hallazgo permitió hipotetizar la posibilidad de una insuficiencia luteal o hipoluteinismo, situación que se confirmaría tras la administración de progesterona micronizada, al observar una normalización en los niveles séricos de $\mathrm{P}_{4}$, la suspensión del profuso sangrado vaginal y la normal evolución de la preñez hasta su término.

Según Lamm y Makloski (2012), la insuficiencia luteal o hipoluteinismo puede ser de base primaria en caso de falla ovárica o secundaria en caso de falla hipofisiaria. Günzel-Apel et al. (2012) señalan que se desconoce la incidencia de hipoluteinismo en poblaciones de perras reproductoras, debido al subdiagnóstico y a la escasez de datos clínicos. Cabe destacar que, en términos generales, la etiología de esta alteración endocrina no aparece bien definida en los textos de especialidad; sin embargo, recientemente se ha descrito la presencia de anticuerpos antiprogesterona que podrían explicar pérdidas gestacionales y acortamientos del ciclo estral (Krachudel et al., 2013).

El diagnóstico de la insuficiencia luteal resulta complicado ya que se requieren muestras de sangre seriadas, prácticamente una vez a la semana, para poder establecer la disminución en los niveles normales de la hormona luteal; es decir, un descenso inferior de $2 \mathrm{ng} / \mathrm{ml}$ (Lamm y Makloski, 2012). El descenso de $\mathrm{P}_{4}$, al ocurrir entre la cuarta y quinta semana de gestación, indicaría hipoluteinismo y puede ser considerado un prerrequisito para comenzar la suplementación hormonal de modo de evitar la pérdida gestacional (Root Kustritz, 2005). En el presente caso, el diagnóstico de hipoluteinismo se realizó empleando un análisis semicuantitativo por ELISA, tal como señala Root-Kustritz (2001). Estos kits son muy exactos en el rango alto de la hormona y son útiles para el diagnóstico de perras que presentan concentraciones de progesterona sérica menores de $5 \mathrm{ng} / \mathrm{ml}$ (Manothaiudom et al, 1995). 
Según Purswell (1991) y Root Kustritz (2001), el hipoluteinismo se puede tratar con $\mathrm{P}_{4}$ natural inyectable. La progesterona oleosa es una preparación inyectable de depósito y que se administra en dosis de $10 \mathrm{mg} / \mathrm{kg}$ cada 48 horas por vía intramuscular (Lamm y Makloski, 2012). Esta $\mathrm{P}_{4}$ se puede medir por análisis con un kit cualitativo comercial (ELISA) o radioinmunoensayo cuantitativo de laboratorio (RIA) para evaluar la suficiencia de la suplementación (Root Kustritz, 2001). $\mathrm{Al}$ considerar las posibilidades terapéuticas en el mercado farmacéutico nacional, se optó por el uso de $\mathrm{P}_{4}$ micronizada de uso humano (al no disponer de la opción inyectable) en dosis de $100 \mathrm{mg} /$ día por 5 días, manejo que permitió normalizar el desarrollo de la preñez y efectivamente incrementar los niveles sanguíneos de $\mathrm{P}_{4}(>5 \mathrm{ng} / \mathrm{ml})$

La progesterona fue administrada hasta aproximadamente los 58 días de gestación, momento a partir del cual se suspende el tratamiento de acuerdo con Root Kustritz (2001), quien señala que la progesterona sérica o la concentración de progestágenos debido a la suplementación declinan dentro de un día de retirado el suplemento oral, y dentro de los tres días después de la última inyección de progesterona oleosa, lo cual permitiría el desarrollo endocrino normal del parto.

\section{Literatura Citada}

1. Concannon P. 2011. Reproductive cycles of the domestic bitch. Anim Reprod Sci 124: 200-210. doi: 10.1016/ j.anireprosci.2010.08.028

2. Günzel-Apel1 A, Urhausen C, Wolf K, Einspanier A, Oei C, Piechotta M. 2012. Serum progesterone in pregnant bitches supplemented with progestin because of expected or suspected luteal insufficiency. Reprod Dom Anim 47(Suppl 6): 55-60. doi: 10.1111/ rda. 12029

3. Hoffmann B, Höveler R, Hasan SH, Failing K. 1992. Ovarian and pituitary function in dogs after hysterectomy. $\mathbf{J}$ Reprod Fertil 96: 837-845. doi: 10.1530/ jrf.0.0960837

4. Johnston S, Kustritz, M, Olson P. 2001. Canine pregnancy. In: Kersey R (ed). Canine and feline theriogenology. $3^{\text {rd }}$ ed. Philadelphia, USA: W.B. Saunders. p 6699.

5. Kowalewski M. 2014. Luteal regression vs. prepartum luteolysis: Regulatory mechanisms governing canine corpus luteum function. Reprod Biol 14: 89-102. doi: 10.1016/j.repbio.2013.11.004

6. Krachudel J, Bondzio A, Einspanier $R$, Einspanier A, Gottschalk $J$, Kuechenmeister $U$, Muennich A. 2013. Luteal insufficiency in bitches as a consequence of an autoimmune response against progesterone? Theriogenology 79: 1278-1283. doi: 10.1016/ j.theriogenology.2013.02.025

7. Lamm C, Makloski C. 2012. Current advances in gestation and parturition in cats and dogs. Vet Clin North Am Small Anim Pract 42: 445-456. doi: 10.1016/ j.cvsm.2012.01.010

8. Linde-Forsberg C. 2010. Pregnancy diagnosis, normal pregnancy and parturition in the bitch. In: England G, von Heimendahl A (eds). BSAVA Manual of canine and feline reproduction and neonatology. $2^{\text {nd }}$ ed. England: BSAVA. p. 89-97.

9. Manothaiudom K, Johnston S, Hegstad R. 1995. Evaluation of the Icagen-Target canine ovulation timing diagnostic test in detecting canine plasma progesterone concentrations. J Am Anim Hosp Assoc 31: 57-64.

10. Purswell B. 1991. Management of apparent luteal insufficiency in a bitch. $\mathbf{J}$ Am Vet Med Assoc 199: 902-903.

11. Root-Kustritz M. 2001. Use of commercial luteinizing hormone and progesterone assay kits in canine breeding management. In: Concannon PW, England G, Verstegen J (eds). Recent advances in small animal reproduction. Ithaca: International 


\section{A. Sánchez}

Veterinary Information Service (www.ivis.org).

12. Root Kustritz, M. 2005. Pregnancy diagnosis and abnormalities of pregnancy in dogs. Theriogenology 64: 755-765. doi: 10.1016/j.theriogenology.2005.05.024
13. Verstegen J, Dhaliwal G, VerstegenOnclin K. 2008. Canine and feline pregnancy loss due to viral and noninfectious causes: a review. Theriogenology 70: 304-319. doi: 10.1016/ j.theriogenology.2008.05.035 